\title{
ACERCA DE LAS BASES TEÓRICAS, FILOSÓFICAS, EPISTEMOLÓGICAS DE LA INVESTIGACIÓN CIENTÍFICA - EL CASO DE LA ENFERMERÍA ${ }^{1}$
}

Vilma de Carvalho ${ }^{2}$

Carvalho V. Acerca de las bases teóricas, filosóficas, epistemológicas de la investigación científica - el caso de la enfermería. Rev Latino-am Enfermagem 2003 novembro-dezembro; 11(6):807-15.

En este trabajo, la discusión es (como en el título) acerca de las bases teóricas, filosóficas, epistemológicas de la investigación científica - el caso de la enfermería. La autora toma de partida las cuestiones fundamentales de la Enfermería - ciencia y arte. Pero no solo en el interés de la profesión como práctica social, sino tambien como práctica de la investigación y de la construcción científica. La Enfermería es así comprendida como "ciencia-en-contrucción", y como un conocimento específico aunque impreciso, sin embargo en vias de tornarse científico por la confiabilidad de los resultados y por sus evidencias. Además, es una ciencia que se justifica no por la precisión sino por la necesidad misma de buscar respuestas para la posibilidad de elevarse al alcance epistemológico de los resultados de la investigación en enfermería - su saber y su quehacer. Y a seguir el enfasis radica en la cuestión del cuidado que debe ser el punto crucial de la reflexión, y tambien el punto de interés mayor para las investigaciones en enfermería.

DESCRIPTORES: investigación en salud; enfermería; bases teóricas/filosóficas/ epistemológicas; investigación en enfermería

\section{ABOUT THE THEORETIC, PHILOSOPHICAL, EPISTEMOLOGICAL BASES} OF SCIENTIFIC RESEARCH - THE CASE OF NURSING

As indicated by the title, this paper discusses the theoretic, philosophical, epistemological basis of scientific research the case of nursing. The author points out the fundamental questions in nursing - science and art. Nursing is considered both as a social practice and as a profession that encompasses scientific practice and the construction of knowledge. Thus, Nursing is understood as a "science under construction" or as specific knowledge, even if it is imprecise and, therefore, on its way to become scientific due to its evidences. In other words, knowledge that is justified not by total precision, on the contrary, by the need to find answers to the question about raising the epistemological reach of nursing research results - its knowing and knowing what to do. The discussion emphasizes the crucial nature of nursing care.

DESCRIPTORS: health research; nursing; theoretic/philosophical/epistemological basis; nursing research

\section{ACERCA DAS BASES TEÓRICAS, FILOSÓFICAS, EPISTEMOLÓGICAS DA} INVESTIGAÇÃO CIENTÍFICA - O CASO DA ENFERMAGEM

Neste trabalho a discussão é desenvolvida, como no título, acerca das bases teóricas, filosóficas, epistemológicas, da investigação científica - o caso da enfermagem. A autora toma de partida as questões fundamentais da Enfermagem ciência e arte. Não apenas é visado o interesse da profissão como prática social, mas também o interesse profissional como prática da pesquisa e da construção científica. A Enfermagem é, assim, compreendida como "ciência em construção" ou como um conhecimento específico, ainda que impreciso e, portanto, em vias de tornar-se científico por suas evidências. Ou seja, um conhecimento que se justifica não por absoluta precisão, ao contrário, pela necessidade mesma de buscar respostas para a questão de elevar o alcance epistemológico dos resultados das pesquisas/investigações na enfermagem - seu saber e seu que-fazer. A ênfase da discussão repousa na questão do cuidado prestado como ponto crucial, não apenas para reflexão, mas, também, como ponto de interesse maior para a pesquisa na enfermagem.

DESCRITORES: investigação em saúde; enfermagem, bases teóricas/filosóficas/ epistemológicas; pesquisa em enfermagem

\footnotetext{
${ }^{1}$ Curso/Encuentro Internacional de Investigación en Salud. Un Programa Científico del Colégio Nacional de Enfermeros del Perú. Ciudad de Lima, Junio $2001 ;{ }^{2}$ Enfermera, Doctora/Docente Libre, Profesora Titular de la Escuela de Enfermería Anna Nery de la Universidad del Brasil (Universidade Federal do Rio de Janeiro - UFRJ); Licenciada en Filosofia; Pesquisador del Consejo Nacional de Desarrollo Científico y Tecnológico - (CNPq/ Brasil); e-mail: decarvalho@eean.ufrj.br
} 
DE LA NECESIDAD DE UN PUNTO DE VISTA

Profesionalmente, yo soy enfermera, antes de cualquier otra cosa. Entonces necesito hablar en nombre de la Enfermería. Mismo en atención a la Filosofía, que es la otra profesión que yo tengo, mis palabras son dirigidas a los enfermeros. Los profesionales de otras áreas de conocimiento que sean investigadores, aúnque ellos sean del área de la salud (posiblemente interesados en el tema) necesitan disculparme. Pues, lo mucho (o lo poco?) que yo he aprendido sobre este asunto en mi vida de profesora de Filosofía y Epistemología, ha sucedido solamente por motivo de la necesidad de intercambiar con mis colegas y estudiantes las preocupaciones con las cuestiones fundamentales de la Enfermería - ciencia y arte. Primero, por los motivos de la práctica profesional, además, en razón de los requerimientos de la investigación científica y por el interés de la profesión. Un asunto historicamente más bien sucedido y ya resaltado (se puede decir) en muchas partes del mundo, desde los anõs del último cuarto del Siglo XX.

En nuestros dias, en este inicio de Siglo XXI (III Milenio de la Era Cristiana), la principal cuestión de entre todas las de interés de los enfermeros se ha tornado, por supuesto, la investigación científica. En realidad, por fuerza del progreso científico y tecnológico. Mas también por la terrible presión en que se ha tornado la práctica académica en el campo de las actividades universitárias, y porque, en consecuencia, todos los profesores tienen que producir conocimientos y comunicar los trabajos que resultan de sus estudios e investigaciones. Pero, es necesário decir que la posible riqueza de mis experiencias con la investigación científica, en gran parte de lo que yo tengo aprendido con este asunto, es el fruto más significativo de la práctica con las orientaciones de las disertaciones y tesis de los estudiantes. en el Programa de Post-Grado de la Escuela de Enfermería Anna Nery - Universidad Federal de Río de Janeiro (UFRJ). Lo que ha sucedido, en realidad, desde los años 70 con la implementación del Curso de Maestría en 1972, y posteriormente con el Curso de Doctorado en 1989.

Así, pues, yo siempre comienzo mis clases con la idea de que la investigación no es posible sin que se tenga en cuenta lo que hay de subyacente a nuestras aspiraciones o a nuestra imperiosa voluntad de conocer y que ha impulsionado nuestras interrogantes sobre los problemas de la profesión. El progreso del conocimiento y nuestra necesidad de conocer es, además, el primordial motivo de toda investigación. Todavía, con nuestras preguntas sobre la naturaleza de las cosas que deseamos investigar, hay siempre asociadas muchas dudas y dificultades. Hay mucha relevancia en la postura intelectual y en la intencionalidad del investigador, y así, también, en la especificidad del tema u objeto que se quiere estudiar y conocer. En general, si se quiere conocer mejor una profesión o una disciplina de estudio como la nuestra - la Enfermería - sus aspectos más distintos, o bien más particulares, son muy importantes y hay que saber de que punto se puede comenzar con los cuestionamientos. Yo quiero decir, en donde se encuentra la puerta de entrada para desarrollar las búquedas de interés de la ciencia. La salida nos lleva a la meta de los resultados Hay muchas direcciones o caminos, por los cuales debemos desarrollar los procedimientos de la investigación para alcanzar las respuestas para nuestros objetivos.

Para eso necesitamos, también, de dar una mayor atención a los trazos más importantes de la ciencia (re)conocida. El primero y más saliente es el control práctico de la naturaleza; el segundo es la sistemática objetiva; y el tercero es el método de la investigación. Como dice Nagel ${ }^{(1)}$, necesitamos poner atención en la lógica y en las implicaciones que esos trazos resultan para nuestras investigaciones. Principalmente si deseamos provocar los cambios en la realidad de la práctica. En consecuencia, en el ámbito de la ciencia, "la teoría es el aspecto de mayor importancia”(2). La teoría es una forma de explicación, en el plan del conocimiento, y es la esfera del sujeto cognosciente (o del investigador). Las bases teóricas, por supuesto, permiten tornar claras las dificultades de los temas, de los detalles técnicos y de los aspectos más distintos de los caminos metodológicos, bien como de la propia estructura de la investigación que se pueda procesar.

Con razón se puede decir cual es lo específico de una profesión de nivel académico y de sus intereses (como ejemplo la investigación), pues, "el académico es el teórico"(3). Así, para tratarse de la investigación, sea como un asunto de estudio o de procedimiento para alcanzar el conocimiento, hay necesidad de disponerse de "la teoría". De esta nuestra profesión, solamente se puede decir que ella es una ciencia en construcción, o como bien afirma Moles ${ }^{(4)}$, "una ciencia-en-via-de-hacerse" y, por lo tanto, "una de las ciencias del impreciso". Como ya sabemos la meta de la ciencia es la precisión. Y en 
nuestra "arte de cuidar en enfermería", nosotros los enfermeros actuamos en las bases de una práctica científica. Pero, los resultados de nuestras investigaciones aún no se pueden resaltar, en su totalidad, con una absoluta "precisión", la cual es requisito de la ciencia. Mas precisamos comprender que los requisitos científicos conclaman a la distinción y al alcance epistemológico. Así, la pregunta que se coloca, para nuestra reflexión, es la seguiente: Que hay que hacer para elevar los resultados de la investigación en enfermería al alcance epistemológico pretendido?

No es tán simple tratar de las cosas en relación a las explicaciones de nuestras investigaciones. Pienso que es necesário para los enfermeros, que ellos mismos puedan comprender y asumir la posición intelectual, de que algunas bases teóricas son indispensables para enraizar el pensamiento sobre la enfermería y, principalmente, sobre el pensamiento escencial de base para la investigación científica a ser procesada. Entonces, como yo comprendo, que nuestra primera reflexión, sobretodo, es con la idea de la especificidad del objeto de nuestro mayor interés - el cuidado que es prestado [0 que es ofertado] a los clientes. Por lo menos en la práctica asistencial de la salud, "el cuidado de enfermería" debería ser el punto crucial de la atención que es dada a los clientes. Y, así, él pueda merecer el indicativo de ser el principal objeto de estudio, como el más precioso punto para la investigación de entre muchas que hay y que están en proceso para edificar la construcción científica. Todos los profesionales de enfermería, en efecto, en la totalidad de la práctica, son los interesados en los cuidados de los clientes que necesitan de su atención y de los resultados de sus investigaciones; hasta porque eses resultados pueden servir a la promoción y a la implementación de mejores cuidados en enfermeria.

Mi posición personal es la de que entre todos los aspectos de las investigaciones relacionadas con la construcción científica, en nuestra área profesional, tienen que ver con el significado y con la singularidad de los cuidados de enfermería dados a los clientes. Y eso en la valoración universal, o sea, sin dependencia de cualquier sector de actuación profesional, o cualquiera que sea la finalidad de la especialidad de los enfermeros. En mi punto de vista, sin atención a esta cuestión de alta relevancia no se debería pensar ni hablar de la construcción cientifica para la enfermería. Esto, sin querer asumir una posición de máxima excludencia. Hasta porque, yo estoy cierta de que aún tenemos extrema necesidad de muchas y diversas investigaciones para aclarar la historia de nuestro saber $y$ quehacer. Y así hay mucho que investigar en relación a la propia História de la Enfermería. Pues comprendo, que nuestra história es parte integrante de nuestra identidad profesional. En este aspecto, las bases explicativas de que necesitamos son, también, indispensables a las investigaciones que se refieren al contexto más próximo o más amplio. Así, mis palabras deben señalar en relación "al contexto histórico" - en consistencia con la marca paradigmática de la profesión-y "al contexto social", cuya coherencia tiene relación con la posición profesional de los enfermeros, en el área de la salud, de la educación, y en el mundo en que vivimos.

Sin embargo estamos preocupados con la construcción cientifica, por lo que las investigaciones que tratan de los cuidados, de entre otros tantos asuntos, deben merecer una atención especial de los enfermeros. Los cuidados de enfermería son, en efecto, la raison d'étre (la razón de ser), de nuestro saber y quehacer. Y para la sobrevivencia de este nuestro "saber y quehacer", los cuidados a los clientes sirven para explicar el propósito de la enfermería y de sus modelos de cuidar y de formar (preparar educacionalmente) el personal profesional. La investigación, así como pienso, es el proceso científico y el mejor estilo sistemático de pensar sobre los cuidados de enfermería. La idea no es tán originalmente fruto de mi propia creación ${ }^{(5)}$. Además, quiero reportarme a Florence Nightingale, la fundadora de la Enfermería Moderna que en sus formulaciones explicativas en el best seller con el título de "Notes on Nursing: what it is and what it is not"(6). Como ya sabemos, en su magnífica exposición sobre el arte de enfermería - una ineludible "arte de cuidar" - son "los cuidados a los clientes" es el aspecto que ella resalta como siendo el centro del mas elevado interés. Y asumo, por cierto, que Nightingale es la principal teórica de nuestra profesión, pienso que con su singular y extremada sabiduría, ella indica los princípios fundamentales de esta nuestra singular arte como el punto esencial de sus explicaciones $^{(7)}$. Su mejor crítica Seymour ${ }^{(8)}$, afirma que "los princípios son básicos - [la idea teórica de ellos] como el suporte al proceso reparativo de las enfermedades, y a la perspectiva de restauración de la propia naturaleza del hombre". En consecuencia, ahí está el crivo de las raízes de origen, o el punto de emergencia primária de las bases teóricas que nos permiten comprender y explicar la Enfermería Moderna. 
De esta manera, nuestras bases teóricas comienzan con "la idea de los princípios nightingaleanos". En toda mi vida profesional, he comprendido que los principios de nuestra arte de cuidar, sin ninguna duda, se constituyen en nuestro primordial marco de referencia. Si se quiere, los princípios son nuestro punto de partida con respecto a los fundamentos de la investigación científica de nuestro interés. Así, desde el punto de vista y sobre el tema que se ha designado Las Bases Teóricas, Filosóficas, Epistemológicas de la Investigación Científica, es un asunto de interés para todos los investigadores en nuestros dias, pero sólo puedo hablar con el propósito y firmeza de las palabras direccionando mi discurso sobre el caso de la enfermería.

\section{LO QUE HAY PARA SABER Y HACER EN LA INVESTIGACIÓN CIENTÍFICA}

Lo que nos remite al saber y hacer en cuanto a la investigación científica, no es tán simple de explicar, por lo menos con respecto al área de la enfermería. Pero, de modo particular, soy, de hecho un tanto eclética en mi postura intelectual y académica. No hay como simplificar mis palabras. Hay mucho que estudiar y practicar en el plano de las propias investigaciones. Así, pienso que los cuadros de referencia y los encuadramientos topológicos - teóricos, técnicos (profesional), metodológicos, y epistemológicos - "del objeto" a ser investigado son más que necesários ${ }^{(9)}$. Pienso, también, que un método de investigación es bueno desde que sea aplicable a nuestro propósito de participar en la construcción científica de nuestro interés. Además, con la cantidad y la calidad de los resultados de que la enfermería necesita. Y si es así como pienso, aunque la investigación científica y sus metodologías no sean completamente dominadas, por los enfermeros, hay que dar atención a los trazos distintivos de la ciencia ${ }^{(1)}$. Creo, pues, que precisamos tener en cuenta la necesidad de muchas investigaciones que puedan resaltar los resultados que sean más adecuados a las caracteristicas de lo que se pueda aceptar como ciencia.

Por cierto, en la trayectoria de evolución de más de 140 años de La História de la Enfermería Moderna, no solamente Florence Nightingale ha presentado "las bases teóricas" que interesan a nuestras investigaciones. El tiempo para esta exposición es más que reducido. Pero, debo decir que la construcción científica del cual tenemos conocimeinto y a la quel debemos nuestras consideraciones, se enrriqueció desde los años 70 , principalmente en los Estados Unidos de América, donde hay una buena cantidad de formulaciones teóricas que todos nosotros podemos y debemos consultar ${ }^{(10)}$. La literatura de enfermería que se puede adoptar como referencial teórico es, inclusive, bien amplia. Yo no pretendo tratar de este aspecto en sus detalles. Es mi pensamiento, todavia, que las teorías de enfermería, aúnque buenas concepciones, son tentativas de conocer sobre el todo que a nosotros nos interesa [saber y hacer] en relación a las actividades de los enfermeros. En realidad, las teorías son tentativas de explicación y sirven con sus conceptos esenciales para afirmar el pensamiento. Y sirven, también, como ejemplos de lo que yo comprendo como la teorización sobre la práctica. En su mayoria, las mismas tratan de los cuidados de enfermería a los clientes. De hecho, aunque sean muy buenas para ancorar los estudios y las investigaciones, no ofrecen, por si, conditio sine qua non para las aplicaciones en la práctica asistencial. Además, (esto me parece), aún no están causando, o no provocan, el impacto esperado en el conocimiento y en lo social. Talvez porque están necesitando de nuestras conjeturas y refutaciones ${ }^{(11)}$, y necesitando tambien de los requisitos de falseabilidad y verificabilidad ${ }^{(12)}$.

Solamente para suscribir este aspecto del asunto, quiero decir que si precisamos de los resultados del nivel del conocimiento confiable ${ }^{(13)}$, tenemos que exponer "las teorias de enfermería" a los criterios y reglas de las pruebas de la ciencia. Las investigaciones "teóricas" en el Brasil, y también (yo creo) en esta parte de Latino América, son aún como que casuales, si es que así se puede considerar, y necesitan de pruebas o de experiencias más bien instruídas de acuerdo con el control práctico de la naturaleza ${ }^{(1)}$. Si hay muchas dificultades para superar, tenemos que considerar que las investigaciones con bases en nuestras propias teorias no son tán repetidas o frecuentes y así continuan en una situación de aquellas del tipo primera experiencia, o experiencia imediata. En las palabras de Bachelard ${ }^{(14)}$, por lo poco que sea "la primera experiencia", casi siempre resulta en sérios obstáculos epistemológicos que no confieren a la construcción científica y a las investigaciones procesadas el alcance de la objetividad más estricta o la cientificidad de que nuestra profesión necesita.

No quiero decir con esas palabras que soy una 
"apologista de la precisión", ni tampoco que soy la defensora radical de los resultados de las investigaciones basadas en explicaciones del estilo de la Teoria de los Objetos $^{(15)}$. También, no estoy para perder mi tiempo con otras tantas teorias que aseguran las profesiones en la búsqueda de la cientificidad como la única meta. Mas pienso que es necesario prestar atención a algunas teorias que sean escenciales al trabajo del investigador interesado en la construcción de la ciencia. Creo, también, que la objetividad es una meta distintiva de las investigaciones cientificas. Y si deseamos que nuestra profesión alcance el patamar de las ciencias (re)conocidas, entonces hay que dar atención a las bases teóricas - Ciencia, Filosofía, Epistemología, y Enfermería. En cuanto a esta última, con particularidad, porque pienso y defiendo mi posición profesional en relación a la investigación científica, de que necesitamos de mucho apoyo teórico y de otro tanto de los procedimientos metodológicos.

\section{LO MÁS IMPORTANTE SOBRE LAS BASES DE LA INVESTIGACIÓN CIENTÍFICA}

Mis conocimientos no son tán profundos como me gustaría que fueran y, despues, es muy difícil la reductibilidad conceptual en un tema tan complejo. Pero, comprendo que de este asunto sobre las bases de la investigación cientifica, en una circunstancia particular o en otra cualquiera, nadie se escapa de una opción bien simple encuanto a la expectativa general con la ciencia. Esto es, o se consideran las teorias que se reportan a las ciencias puras con atención a los paradigmas de la Física y de las ciencias matemáticas, o se considera los modelos teóricos de las ciencias del espírito, las cuales son ya más bien conocidas como las ciencias humanas y sociales. Como siempre afirmo, en relación al teórico, mi posición intelectual es un tanto eclética y, encuanto al método, me complace mucho un cierto estilo artesanal de hacer ciencia(16) $^{(16}$ en este sentido, encuanto a los procedimientos metodológicos, me considero un tanto valetudinária o de un estilo como que anárquico, como quiere Feyerabend ${ }^{(17)}$; esto es, cualquier que sea el método, pienso que puede ser bueno desde que sea aplicable al objeto de estudio.

Así, pues, pienso que es mejor no tener una postura hermética o excludente. Pero esto no quiero que sirva de prescripción. Mismo porque yo creo que los problemas de interés para el saber de enfermería ${ }^{(18)}$, tanto cuanto las investigaciones para la construcción cientifica no pueden ser dejados al acaso o un segundo plano. La literatura en el Brasil y también en otros países de Latino América, es suficiente para apoyar e instruir las investigaciones científicas. Para la enfermería precisamos tanto de las bases y métodos de las ciencias nomotéticas (las ciencias de la naturaleza y de las leyes científicas), como necesitamos de los requisitos y reglas de las ciencias ideográficas (las que tratan de las representaciones de las ideas y del análisis del lenguaje).

Observandose por el ángulo de los aspectos más generales, y en relación a la Filosofía, pienso que el punto de partida (para el investigador) es una cuestión de posición personal y de postura intelectual. Esto es, si se parte de un punto de interés del idealismo o del otro punto que es el realismo se sigue en un caso o en el otro, la dirección más clásica en el plano de la búsqueda del conocimiento. Si la preferencia del camino está con la modernidad de la ciencia, las filosofías de apoyo al conocimiento son el racionalismo y el empirismo. Y pienso que para hablar de un punto de vista filosófico y epistemológico, "la dificultad mayor de los investigadores, en el caso particular de la enfermería, está en preferirse de entre muchos un cierto médodo y no otro, sin saber, con efecto, cual es el más adecuado, tampoco ellos saben decidir, por cierto, cual es la mejor teoría de apoyo o de sustentación que debe merecer el mayor peso en sus estudios"(19) ${ }^{\text {(19) }} \mathrm{Y}$ esta es una posición personal que asumo frente a la práctica académica en el ámbito de la investigación científica. Entonces, pienso que es preciso saber decidir de donde podemos asumir nuestro punto de partida (la teoría) y de cual camino (el método) nos parece el mejor para la investigación del objeto de estudio de nuestro interés. Esta es una cuestión, por lo tanto, que precisa de nuestra (re)consideración.

\section{LA ENFERMERÍA EN LA CRISIS CIENTÍFICA Y EPISTEMOLÓGICA DE NUESTRO TIEMPO}

Durante más de trescientos años, desde las concepciones de Galileo, Newton, Copérnico, y Descartes, las bases explicativas de "las ciencias puras y los princípios del método científico" orientaron e impulsionaran las investigaciones y la construcción científica en toda la Época Moderna. Al final del Siglo XIX y el início del Siglo 
$\mathrm{XX}$, fueron muy grandes las mutaciones en el ámbito de las ciencias, principalmente en razón de la crisis epistemológica de la Física señalada por la Teoria de la Relatividad y por las proposiciones de la nueva Física Cuántica. Una crisis mas séria (talvez) que la anterior desencadenada con la Renascencia y todo el proceso histórico de la Modernidad. Una crisis demarcada por los cuestionamientos filosóficos con pertinencia a las posibilidades del conocimiento humano, al espacio epistemológico de las investigaciones y al alcance o límites de los resultados obtenidos para las búsquedas direccionadas, principalmente, para la construcción científica. La Filosofia de la Ciencia, ahora más bien conocida como Epistemología, alcanza gran relevancia para las ideas y concepciones de la construcción científica. $Y$ con ella ciertos pensadores como Bachelard ${ }^{(14,20)}$, Foucault ${ }^{(21-23)}$, Kuhn ${ }^{(24)}$, Popper ${ }^{(12)}$, Piaget ${ }^{(25)}$, y otros más dedicados a las cuestiones epistemológicas y que resaltan, la Filosofia como crítica del conocimiento y, en consecuencia, crítica de la investigación científica. En este sentido, hay que conocer otros pensadores interesados en los estudios epistemológicos y que merecen nuestras lecturas y consideraciones ${ }^{(26-29)}$.

Sin embargo las investigaciones científicas aún continuan con requerimientos estrictos y se puede decir, mismo cuando "las ciencias del espírito" ya empezaron a ejercer influencias en los estudios e investigaciones que tratan de los intereses o de los aspectos de la dimensión humana y social. Y la enfermería no se queda afuera. Con la velocidad de los cambios, los enfoques teóricos se enriquecen, el método de la investigación amplía sus posibilidades y surgen nuevos procedimientos, algunos de naturaleza filosófica - fenomenología, dialéctica materialista, filosofía de análise, semiótica del lenguaje, y otras - para impulsionar las investigaciones científicas.

Entonces se puede decir, que el último siglo, fué de gran impacto para el conocimiento humano y para la construcción de las ciencias con enfoque en lo social. Todas las profesiones del área de la salud y sus investigaciones sufren de las mismas influencias, inclusive la enfermería. Además, si de la Filosofía se puede hablar con cierta amplitud de visión, lo mismo no sucede con la Epistemología. Mismo siendo esta comprendida como la Filosofia de la Ciencia. Los conceptos o constructos epistemológicos no son tán facilmente comprendidos y aplicables a los temas o problemas de la investigación en enfermería ${ }^{(19)}$. En mi situación particular, los conocimientos fundamentales de la Filosofía y los asuntos de la
Epistemología, con sus implicaciones para la práctica de la investigación, son parte de mis actividades de profesora por la necesidad misma de la enseñanza en las clases o de las orientaciones a los estudiantes. Entonces veamos como este asunto se ha desarrollado en mis actividades.

Como comprendo, hay que tener en cuenta que la investigación es, para la enfermería, una necesidad de construción científica y de contribución a los adelantos de la práctica pedagógica y a los de la asistencia. Esto es un reto propio de nuestros tiempos. Los adelantos del progreso científico hacen una presión muy fuerte sobre el proprio conocimiento técnico-profesional, no sólo por la velocidad de los cambios, sino por la obsolescencia o caducidad de los instrumentos que sirven a todos los estilos de la labor humano. Casi en todo momento, nuevos artefactos ejercen una diferente demanda a nuestras destrezas y habillidades. Hay como que un verdadero y continuado reto (permanente?) en oposición al status y a las posibilidades de todas las profesiones, principalmente de las que están situadas en las áreas de educación y de salud. Entonces para los enfermeros no hay como escapar. Aúnque ellos no sean investigadores expertos, los que asumen las posiciones del liderazgo necesitan de instrumental de apoyo y de saber utilizar los relatórios y datos de las investigaciones. Ellos necesitan saber como hacer opción entre los resultados que tengan más importancia para cambiar la práctica de la profesión. Esta, pues, es una razón de peso para superar los retos en la vida profesional, y se puede decir una razón más que suficiente de sobrevida para los enfermeros en los escenários diversos del área de la salud. Necesito repetir que la mayor dificultad es que ellos deben saber con propiedad, "como identificar y estudiar los problemas que sean posibles de investigación, así como saber decidir sobre la toma de posición en relación a las teorías que merecen el mayor peso"(19). Entonces, nuevamente, surge la cuestión de las bases explicativas que puedan respaldar o responder por las investigaciones en enfermería.

Esta cuestión de la investigación científica no es tán sólo un apelo, una simple demanda, o un reto típico del acto de conocer. Mas que una cuestión, en efecto, ella es como que un imperativo de nuestro tiempo. En el Brasil, esta cuestión se ha colocado para nosotros, los enfermeros, como una razón incuestionable para que se haya cambiado los Currículos del Prégrado en Enfermería (MEC: a. - CFE Parecer 163/72; b. - Resolución 04/72; y c. - Parecer $314 / 94$ y Portaria 1721/94). Los cambios fueron contínuos, desde los años 70 , con radical 
importancia a las nuevas dimensiones de la práctica asistencial en salud, y sobretodo con los requerimientos de nuevas metodologías aplicables a la práctica de cuidar de los clientes en el contexto del proceso de la enseñanzaaprendizaje. La investigación científica, antes de todo se ha hecho imperiosa en el área de la enseñanza desde el Pré-grado, y como un proceso de saber y hacer. Hasta, porque no era más posible esperarse que los enfermeros (unos pocos) se encontrasen con capacidad y competencia para procesar las investigaciones, y esto solamente despues de los Cursos de Pósgrado - Maestría y Doctorado.

El reto es para todos los enfermeros, docentes y asistenciales. El domínio de la investigación científica más que una necesidad es hoy un deber de todos. Hasta porque las agencias de fomento están cobrando nuestras evidencias en los intentos de cientificidad de las investigaciones en enfermería. $Y$ en consecuencia, se expone a una posición de riesgo el proceso de la formación de los enfermeros en el Prégrado, en la Educación Continuada y mismo en el Pósgrado stricto sensu. Y la situación de las Facultades de Enfermería son colocadas en riesgo en el contexto universitário. Entonces la investigación científica se ha tornado un balón de ensayo en tal ebullición que puede explotar con la enseñanza sustantiva de la enfermería y todo lo que se refiera a sus pretensiones científicas.

Esto lo afirmo porque, en el Brasil (y talvez en toda Latinoamérica), es con mucha dificultad que los enfermeros alcanzan la oportunidad de hacer el Pósgrado. Los servicios y órganos empleadores no quieren comprender los objetivos de la educación continuada en enfermería y talvez sea preciso vencer, también, los estereotipos. El patrimonio individual es muy raro, y la ayuda de financiamento para realizar los estudios y proyectos de investigaciones son muy dificiles. En realidad, las agencias de fomento no están de acuerdo en relación a la calidad epistemológica de los resultados de las investigaciones y con la producción intelectual de los enfermeros. Casi siempre, las apreciaciones o las evaluaciones de los consultores ad hoc, en esas agencias, son injustas para las expectativas de los investigadores en enfermería. Talvez porque ellos juzgan que las ideas en las investigaciones no son bien procesadas, en el área de la enfermería. En el pólo opuesto, las reclamaciones de los enfermeros investigadores destacan que siendo la enfermería una "ciencia-en-construcción" sus investigaciones no deben ser evaluadas por los critérios de las ciencias más bien reconocidas.
Si la necesidad de procesar las investigaciones es un reto ineludible para los enfermeros, entonces tenemos que acertar posición e nuestros pasos, con las otras profesiones más bien cientificamente evaluadas, y siempre de acuerdo con los requisitos de la ciencia. Pienso también que debemos nuestra atención a otras buenas razones. La primera, es porque el conocimiento de interés para la ciencia y para la construcción científica nos presiona con la sistemática de la investigación bien de acuerdo con los trazos distintivos de la ciencia ${ }^{(1)}$. Tanto hace que sea en la forma de pensar (saber) sobre las acciones y sobre los cuidados de la enfermería, o mismo que sea por el simple propósito de actuar (quehacer) de una forma y no de otra. Otra razón, porque el alcance de las investigaciones debe corresponder de preferencia, y hasta con extremada relevancia, con la posibilidad de matematización de los resultados.

Particularmente, no soy una investigadora que persigue la precisión de los datos. Pero los teóricos de la Ciencia son los que afirman que el método científico es el único que puede garantizar la determinación de las leyes y de causar el impacto para los resultados de las investigaciones. Entonces nosotros tenemos que buscar la eficacia y la certeza, por lo menos, para nuestros registros e informaciones pertinentes con los resultados obtenidos de nuestras investigaciones sobre los problemas de la práctica. Tal como pienso, el emprendimiento epistemológico precisa culminar, por lo menos, con los núcleos substantivos, con las ideas centrales, bién como con las categorias o tipologias conceptuales, que permitan la clasificación y la nominata de nuestros datos y de todo lo demas que resulte de nuestras investigaciones. La producción científica de los investigadores de enfermería - docentes, asistenciales y estudiantes (Prégrado y Pósgrado) está precisando de reconocimiento. Y el pensamiento resultante de las investigaciones necesita de constituirse como "de calidad" para la edificación de un corpus teórico suficiente y valioso para sustentar la posición profesional, la cual, por si misma, sea la más adecuada a un estatuto de naturaleza epistemológica para la enfermería.

\section{PARA DONDE DIRECCIONAR EL DISCURSO SOBRE LA INVESTIGACIÓN}

Tomando todo lo expuesto como suficiente para una conclusión, pienso que nosotros, los enfermeros 
investigadores o no, tenemos ahora un aspecto significativo muy importante para enraizar el tema que nos congrega y que nos conclama en esta oportunidad. $Y$ esto es el aspecto central de nuestro interés. $Y$ para facilitar la comprensión, la pregunta que se coloca es la siguiente: Como colocar las investagaciones al servicio de la construcción científica que sea de valor para elevar la posición de la enfermería?

En mi opinón personal, no hay una mejor forma de discutir todo este asunto, y en relación al caso de enfermería, sino fuera enfrentando la necesidad de poner la investigación en relación a sus bases de encuadramiento - topólogico y referencial -, a las demandas de la sociedad y de la academia misma. Tengase en cuenta que los fundamentos del conocimiento científico son de tal relevancia para las investigaciones, así como son los fundamentos de la enfermería, y desde que esa sea asumida como una profesión cuya práctica es necesariamente de bases científicas. En este aspecto, debe ternerse nuevamente en consideración las formulaciones explicativas de Florence Nightingale ${ }^{(30)}$. Para mi es muy valioso que se pueda agregar, de hecho, lo que hay de mejor en cuanto al nivel acádemico del pensamiento sobre enfermería. en el contexto de una forma especial de cuidar de la salud de las personas y de los grupos humanos, - como es el caso de la Enfermería - , las bases explicativas de nuestra primera teórica y sus ideas sobre los principios de los cuidados son representativos de un aspecto del cual nosotros podemos denominar a turning point of history ${ }^{(31)}$. O sinó necesito decir que las ideas nightingaleanas tienen su mas elevado valor al que Bachelard $^{(32)}$ refiere como la expresiva "categoria" - el corte epistemológico. Esto, lo afirmo con extrema consideración, lo que no nos es permitido olvidar.

Por cierto, en el plano de las metodologías científicas, con la debida atención a las investigaciones, debo decir, por último pero no por fin, y salvo al mejor juício, que las proposiciones de Florence Nightingale deben ser consideradas y asumidas, sino como las mejores formulaciones de una teoria, entonces con efetividad en cuanto a la significación de una axiomática o como bien refiere Rudner ${ }^{(33)}$, sus principios básicos mismo siendo del tipo de las formulaciones "no-teóricas" ya tienen su valor para la construcción cientifica como la evidencia misma. Y que ellas sean, por así decir, el mas incontestable punto de partida para todo lo sea el pensamiento sobre enfermeria y en consecuencia, una marcante referencia para las investigaciones que tengan como objeto de estudio los cuidados dados a los clientes, tanto cuanto para las que tratan del saber y quehacer en la Enfermería - ciencia y arte.

En suma, principalmente en nuestras situaciones de enfermería, comprendo y creo que la práctica de la investigación científica no es una cuestión de reducir todo nuestro "saber y hacer" a una pura secuencia de operaciones, de procedimientos metodológicos de rigor científico absolutizado, y ni tampoco de seguir los protocolos inmutables de las agencias de fomento. Hay que tener en cuenta que de los conflictos políticos y económico-sociales no es posible escapar. Los retos y embargos para el desarrollo de la investigación científica en enfermería no son casos aislados. Todos los investigadores están sumergidos en la crisis de nuestro tiempo. Pero lo que más importa a nosotros los enfermeros investigadores, pienso que es la convocatória de avanzar. No podemos simplemente caer en la onda de conflictos, de disputas y de retraimientos. Hay que luchar y avanzar. Hay que continuar caminando por las trillas de las investigaciones. Esto es actualmente nuestro mayor reto.

Así, pues, todas las bases y métodos son de capital importancia para la construcción científica de la enfermería. Lo esperado es que su "saber y hacer" se pueda poner de acuerdo con la ciencia, tomada como un proceso lógico, entretanto en contínua construcción. Tengo certeza de que la enfermería es un arte de cuidar de naturaleza muy especial, y que ya está socialmente consolidada. Y, por supuesto, si la enfermería es una ciencia en construcción, entonces sus búsquedas han de encontrarse con las respuestas que resultan en la cantidad y posible matematización de los datos. Pero también, ineludiblemente, no se puede recusar las búsquedas que resultan en respuestas que apelan más a la calidad de los resultados. Y así, en consecuencia, esos últimos resultados necesitan de los tratamientos que sean potencialmente capazes de elevar el valor de la subjetividad, no sólo en cuanto al propio pensamiento sobre la enfermería. Sino sobretodo, en relación al aspecto inherente a la dimensión humana y social de los sujetos, no solo los que ofrecen su atención específica como los que demandan y reciben los cuidados de enfermería a que tienen el derecho inalienable, en cuanto considerado como el inalienable derecho a la salud. Un derecho de todos, y por eso mismo, tán universal cuanto el derecho a la vida y a la felicidad. 


\section{REFERENCIAS BIBLIOGRÁFICAS}

1. Nagel E. In: Morgenbesser S, organizador. Filosofia da Ciência. São Paulo (SP): Cultrix; 1971.

2. Demo P. Teoria - Porque?. In: 1ํ Simpósio Brasileiro de Enfermagem; 1985. Florianópolis (SC): Editora da UFSC; 1985. p.52-68.

3. Pieper J. Que é filosofar/Que é acadêmico. São Paulo (SP): Herder; 1968.

4. Moles AA, Rohmen E. As Ciências do Impreciso. Rio de Janeiro (RJ): Civilização Brasileira; 1995.

5. Chater S. Understanding Research in Nursing. Geneva: WHO; 1975. WHO Offset Publication, 14.

6. Nightingale F. Notes on Nursing: what it is, and what it is not. London: Duckworth; 1970.

7. Carvalho V. A Enfermagem de Saúde Pública como Prática Social: um ponto de vista crítico sobre a formação da enfermeira em nível de Graduação. In: Escola Anna Nery Revista de Enfermagem 1997 julho; 1 (no especial).

8. Seymour L. The Writings of Florence Nightingale (An Oration delivered before the Ninth Congress of the International Council of Nurses, Atlantic City). Florence Nightingale Oration 2; 1947.

9. De Bruyne P, Herman J, De Schoutheete M. Dinâmica da Pesquisa em Ciências Sociais - Os Pólos da prática metodológica. Rio de Janeiro (RJ): Francisco Alves; 1977.

10. George JB. Teorias de Enfermagem - Os Fundamentos para a Prática Profissional. Porto Alegre (RS): Artes Médicas; 1993.

11. Popper KR. El Desarrollo del Conocimiento Cientifico: Conjeturas y refutaciones. Buenos Ayres: Paidos;1965.

12. Popper KR. A Lógica da Pesquisa Científica. São Paulo: Cultrix; 1974

13. Ziman J. O Conhecimento Confiável - uma exploração dos fundamentos para a crença na ciência. Campinas (SP): Papirus; 1996.

14. Bachelard G. A Formação do Espírito Científico. Rio de Janeiro (RJ): Contraponto; 1996.

15. Moles AA. Teoria dos Objetos. Rio de Janeiro (RJ): Tempo Brasileiro; 1981.

16. Becker HS. Métodos de Pesquisa em Ciências Sociais. São Paulo (SP): Hucitec; 1999.

17. Feyerabend P. Contra o Método: esboço de uma teoria anárquica da teoria do conhecimento. Rio de Janeiro (RJ): Francisco Alves; 1989.

18. Almeida MCP, Rocha JSY. O Saber de Enfermagem e sua dimensão prática. São Paulo (SP): Cortez; 1986.

19. Carvalho V. Sobre Construtos Epistemológicos nas Ciências - uma contribuição para a enfermagem. Conferência 11은 Seminário Nacional de Pesquisa em Enfermagem; Belém; PA; Mayo 2001.

20. Bachelard G. O Novo Espírito Científico. Rio de Janeiro (RJ): Tempo Brasileiro; 1968.

21. Foucault M. As Palavras e as Coisas: Uma arqueología das ciências humanas. São Paulo: Martins Fontes; 1966.

22. Foucault M. O Nascimento da Clínica. Rio de Janeiro (RJ): Forense-Universitária; 1977.

23. Foucault M. A Arqueologia do Saber. Rio de Janeiro (RJ): Forense-Universitária; 1987.

24. Kuhn TS. A Estrutura das Revoluções Científicas. São Paulo (SP): Perspectiva; 1982.
25. Piaget J. Psicologia e Epistemologia: Por uma teoria do conhecimento. Rio de Janeiro (RJ): Forense-Universitária; 1978.

26. Gil G, organizador. Filosofia e Epistemologia (Textos de Investigação do G.I.F.E. - 1977/78). Lisboa: Regra do Jogo; 1978.

27. Japiassu H. O Mito da Neutralidade Científica. Rio de Janeiro (RJ): Imago; 1975.

28. Japiassu H. Nascimento e Morte das Ciências Humanas. Rio de Janeiro (RJ): Francisco Alves; 1982.

29. Escobar CH. Epistemologia das Ciências Hoje. Rio de Janeiro (RJ): Pallas; 1975. Série Ciências Humanas - Hoje. 30. Nightingale F. Notas sobre Enfermagem: o que é e o que não é. São Paulo (SP): Cortez/ABEn-CEPEn; 1989.

31. Toynbee AJ. O Desafio do Nosso Tempo. Rio de Janeiro (RJ): Zahar; 1975.

32. Bachelard G. A Epistemologia. São Paulo (SP): Martins Fontes; 1971.

33. Rudner RS. Filosofia da Ciência Social. Rio de Janeiro (RJ): Zahar; 1969. 